\title{
Could you say that was an atrial flutter or not?
}

\author{
Serkan Özsoylu${ }^{1}$, Başak Nur Akyıldız ${ }^{1}$, Adem Dursun ${ }^{1}$, Özge Pamukçu ${ }^{2}$ \\ Divisions of ${ }^{1}$ Pediatric Intensive Care Unit and ${ }^{2}$ Pediatric Cardiology, Department of Pediatrics, Erciyes University Faculty \\ of Medicine, Kayseri, Turkey.E-mail: sozsoylu@hotmail.com \\ Received: 28th December 2017, Revised: 3rd May 2018, Accepted: 10th June 2018
}

SUMMARY: Özsoylu S, Akyıldız BN, Dursun A, Pamukçu Ö. Could you say that was an atrial flutter or not? Turk J Pediatr 2019; 61: 608-610.

\begin{abstract}
Muscle-tremor artefact is a potential cause of misdiagnosis of atrial arrhythmias on electrocardiography (ECG) monitoring. Such errors may lead to inappropriate and potentially dangerous therapies in some patients. We present a case of a patient with uncontrolled seizures whose bedside electrocardiogram monitor analysis appeared to demonstrate atrial flutter with 4:1 conduction through the AV node. The ECG monitor and ECG rhythm strip additionally showed a regular ventricular rate of $94 \mathrm{bpm}$ with an underlying regular 'saw-tooth' baseline. We applied cardioversion to convert to sinus rhythm. Amiodarone was loaded and added to the patients therapy who had atrial flutter after cardioversion. Echocardiogram was performed by a pediatric cardiologist and they noted that the atrial rate and ventricular rate were equal. After this, we began to suspect this situation might be a pseudoflutter due to his muscle contractions. We applied rocuronium to the patient to understand whether this was a pseudo-flutter or not. We saw that the ECG returned to normal sinus rhythm.
\end{abstract}

Physicians especially working in intensive care units should be aware of artifact to avoid unnecessary therapeutic procedures. As Hippocrates said centuries ago "First, do no harm."

Key words: atrial flutter, electrocardiogram, cardioversion.

Electrocardiographic (ECG) artefacts may closely imitate both supraventricular and ventricular tachycardias. $^{1-5}$ Muscle-tremor artefact is a potential cause of misdiagnosis of atrial arrhythmias on ECG monitoring. ${ }^{6}$ Such errors may lead to inappropriate and potentially dangerous therapies in some patients. ${ }^{7}$ This is particularly the case if a single lead, especially a limb lead, is relied on for making a diagnosis of the underlying rhythm. We present a case of a patient with uncontrolled seizures whose bedside electrocardiogram monitor analysis appeared to demonstrate atrial flutter with 4:1 conduction through the AV node.

\section{Case Report}

A 9-month-old boy with a history of motor delay was admitted to the intensive care unit because of uncontrolled seizures.
Despite the use of five anti-epileptic drugs, his convulsions could not be stopped. His medications on admission included fenitoin (5 $\mathrm{mg} / \mathrm{kg})$, levatirasetam $(30 \mathrm{mg} / \mathrm{kg})$, vigabatrin (30 mg/kg), fenobarbital (5 mg/kg), clobazam (1 $\mathrm{mg} / \mathrm{kg}$ ) daily. On physical examination, he appeared microcephalic and had a regular heart rhythm at 140 beats per minute (bpm) and blood pressure of 91/47 mm Hg. Laboratory testing revealed hemoglobin level of $10.2 \mathrm{~g} / \mathrm{dL}$, sodium $137 \mathrm{mEq} / \mathrm{L}$, potassium $4.8 \mathrm{mEq} / \mathrm{L}$, chloride $97 \mathrm{mEq} / \mathrm{L}$, calcium $9.7 \mathrm{mg} / \mathrm{dL}$, serum urea nitrogen $5 \mathrm{mg} / \mathrm{dL}$, creatinine $0.6 \mathrm{mg} / \mathrm{dL}$, and glucose $145 \mathrm{mg} / \mathrm{dL}$. The electrocardiogram showed sinus rhytm at $136 \mathrm{bpm}$. We added midazolam infusion $(0,1$ $\mathrm{mg} / \mathrm{kg} / \mathrm{h}$ ) to treatment because of the ongoing convulsions. For his airway safety, the patient was intubated. 
On the third day we noticed an abnormal rhythm on his bedside ECG monitor. ECG rhythm strip showed a regular ventricular rate of $94 \mathrm{bpm}$ with an underlying regular 'saw-tooth' baseline. Consulation was sought regarding antiarrhythmic therapy for what was thought to be atrial flutter with $4: 1 \mathrm{AV}$ conduction (ventricular rate of $94 \mathrm{bpm}$ ). (Figs 1 and 2) We consulted the pediatric cardiology department and they interpreted the ECG as atrial flutter. They recommended cardioversion to convert to sinus rhythm. Amiodarone was loaded to the patient and added to his therapy who had atrial flutter after cardioversion. Echocardiogram was performed by pediatric cardiologist and they noted that the atrial rate and ventricular rate were equal. After this, we began to suspect this situation might be a pseudo-flutter due to his muscle contractions. We applied rocuronium to the patient to understand whether this was a pseudo-flutter or not. We saw that the ECG returned to normal sinus rhythm (Fig. 3). We stopped his antiarrhythmic therapy. We took informed consent from the parent of the patient.

\section{Discussion}

Typical atrial flutter has a characteristic ECG appearance, classically described as an undulating 'saw-tooth' baseline without isoelectric intervals between flutter waves. ${ }^{3}$ The atrial rate is typically around 250-350 per minute (cycle frequency of $5 \mathrm{~Hz}$ ). With 4:1 conduction through the AV node (i.e., every fourth atrial impulse conducted), this would lead to a ventricular rate of $65-85$ bpm. ${ }^{5}$ The ECG findings in our patient mimicked atrial fibrillation both in frequency and amplitude. Despite the presence of atrial flutter on ECG, his hemodynamics was stable, his pulse was rhythmical, and his blood pressure was normal. However, the consistent ECG findings

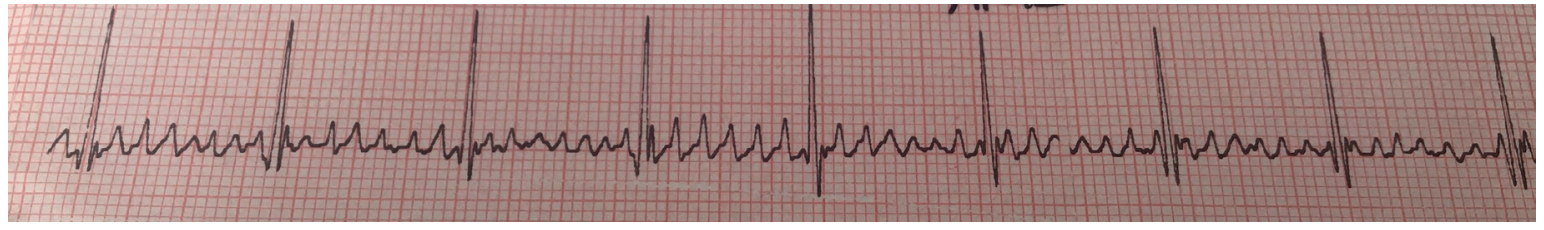

Fig. 1. Short derivation of pseudoflutter.

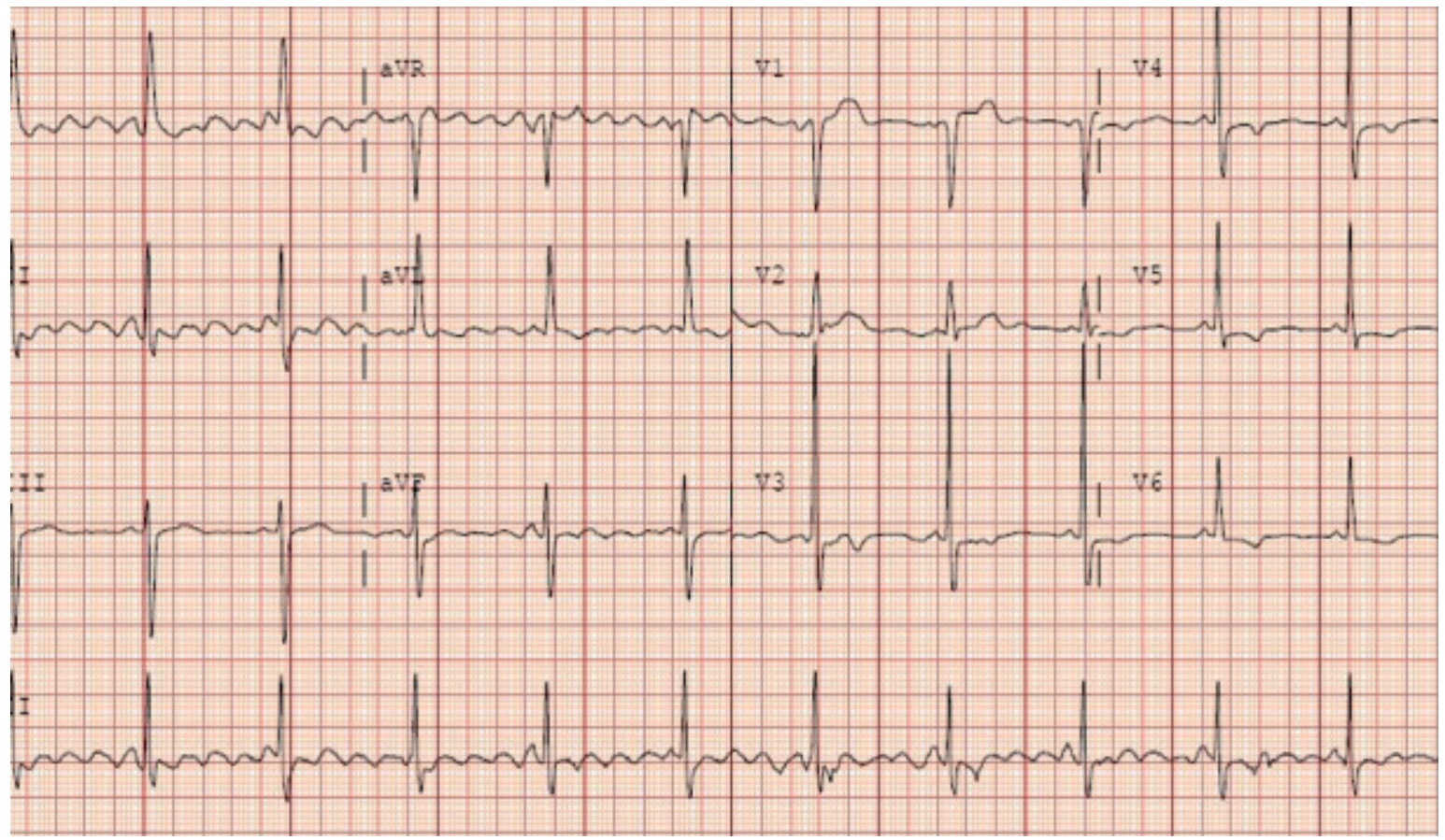

Fig. 2. 12 lead ECG recording of the pseudoflutter. 


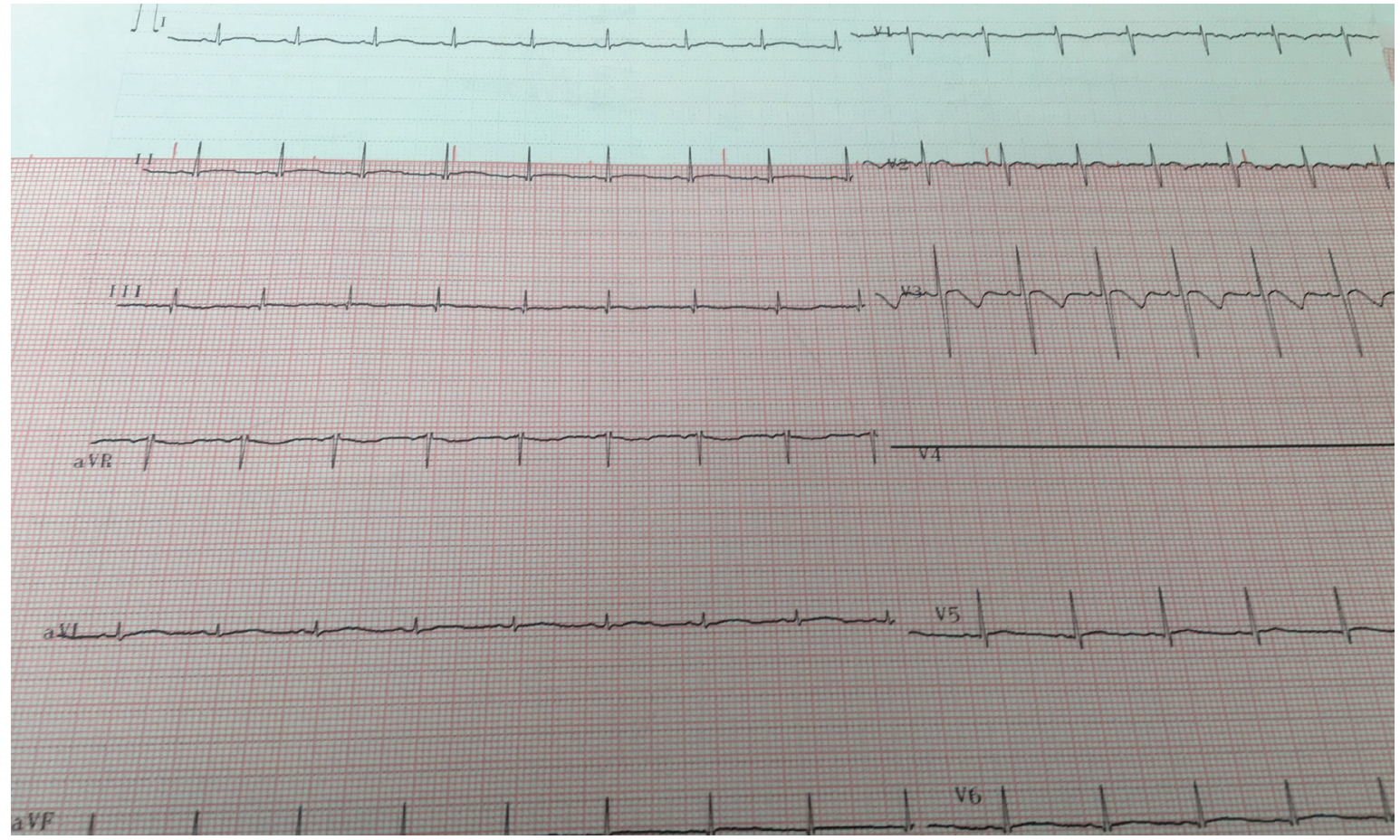

Fig. 3. Normal sinus rhythm after rocuronium.

with flutter waves and the resistant nature of the arrhythmia distracted us. These findings directed us to inappropriate and potentially dangerous therapies.

There is limited information in the literature regarding the clinical implications of misdiagnosing an ECG artefact and this is the first case in an infant in the literature. All cases are in adult age group or neonatal period. The most likely reported causes of ECG artefacts that mimic both supraventricular and ventricular tachycardia are body movements, muscular fasciculations or contractions, tremor in patients with Parkinson's disease, ECMO applications, dialysis treatments. ${ }^{2-7}$

This case shows that the misdiagnosis of electrocardiographic artifact as atrial flutter may lead to unnecessary medication and interventions such as cardioversion and amiodarone. We think that physicians especially those working in intensive care units should be aware of artifacts to avoid unnecessary therapeutic procedures. As Hippocrates said centuries ago "Primum non nocere."

\section{REFERENCES}

1. Krasnow AZ, Bloomfield DK. Artifacts in portable electrocardiographic monitoring. Am Heart J 1976; 91: 349-357.

2. Casta A, McCann ME. Electrocardiographic inscription of diaphragmatic contractions during anaesthesia. Paediatr Anaesth 1999; 9: 175-176.

3. Knight BP, Pelosi F, Michaud GF, Strickberger SA, Morady F. Clinical consequences of electrocardiographic artifact mimicking ventricular tachycardia. N Engl J Med 1999; 341: 1270-1274.

4. Patel S. Electrocardiographic artifact mimicking ventricular tachycardia during high-frequency oscillatory ventilation: A case report. Am J Crit Care 2006; 15: 310-311.

5. Boos CJ, Khan MY, Thorne S. An unusual case of misdiagnosed ventricular tachycardia. Emerg Med J 2008; 25: 173-174.

6. Samaniego NC, Morris F, Brady WJ. Electrocardiographic artefact mimicking arrhythmic change on the ECG. Emerg Med J 2003; 20: 356-357.

7. Finsterer J, Stollberger C, Gatterer E. Oral anticoagulation for ECG tremor artefact simulating atrial fibrillation. Acta Cardiol 2003; 58: 425-429. 